\title{
Application of imaging in lymphedema surgical therapies
}

\author{
Xingyi Du, Chunjun Liu \\ Plastic Surgery Hospital (Institute), Chinese Academy of Medical Sciences, Peking Union Medical College, Beijing 100043, China \\ Contributions: (I) Conception and design: All authors; (II) Administrative support: C Liu; (III) Provision of study materials or patients: X Du; (IV) \\ Collection and assembly of data: X Du; (V) Data analysis and interpretation: All authors; (VI) Manuscript writing: All authors; (VII) Final approval of \\ manuscript: All authors. \\ Correspondence to: Chunjun Liu, MD. Plastic Surgery Hospital (Institute), Chinese Academy of Medical Sciences, Peking Union Medical College, \\ No.33 Badachu Road, Shijingshan District, Beijing 100043, China. Email: Lancet007@126.com.
}

\begin{abstract}
Lymphedema is a chronic, progressive disease caused by primary or secondary reasons. It is currently uncurable and conservative compression therapy is generally applied. Lymphovenous anastomosis and vascularized lymph node transfer (VLNT) are two main surgical treatment that are used in addition to conservative therapy. Lymphovenous anastomosis involves the anastomosing remaining functional lymphatic vessels to vein. When the lymphatic vessels are greatly damaged and in no case can they be used for anastomosis, VLNT provide the affected area with lymph nodes from elsewhere to restore the drainage function. During all these procedures, a clear image to identify related lymphatic structures and venous vessels can be greatly useful for preoperative planning, intraoperative navigation, and postoperative evaluation. Lymphoscintigraphy used to be the gold standard in evaluating lymphedema and mapping lymphatic systems. But due to the downside of radiation, invasive operation and complication, other modalities are gaining attention. In this article, we reviewed the application of Indocyanine green (ICG) lymphography, ultrasound, magnetic resonance lymphography (MRL), and single-photon emission computed tomography-computed tomography (SPECT-CT) in the field of surgical therapy in lymphedema.
\end{abstract}

Keywords: Indocyanine green (ICG); lymphedema; magnetic resonance imaging; single-photon emission computed tomography-computed tomography (SPECT-CT); ultrasound

Submitted Nov 19, 2019. Accepted for publication Feb 27, 2020.

doi: $10.21037 /$ gs.2020.03.24

View this article at: http://dx.doi.org/10.21037/gs.2020.03.24

\section{Introduction}

Lymphedema is a chronic, progressive disease caused either by congenital lymphatic malformation or secondary destruction to the lymphatic channels. Oncologic treatment is the main cause of secondary lymphedema and it often results in extremity pain, swelling, infections, restrictions in range of motion, and aesthetic disturbances, leading to a decrease in patients' quality of life (1).

Currently, there is no curative treatment for lymphedema. Combination use of manual lymph drainage, compression, exercise, and skin care called complete decongestive therapy (CDT) are recommended. However, for those who do not respond to these therapies, surgical interventions are indicated. Lymphovenous anastomosis
(LVA) improves lymphedema by linking lymphatic collecting vessels (LCVs) with vein. Vascularized lymph node transfer (VLNT) involves transferring lymph nodes into the affected limb to restore the drainage function $(2,3)$. In either case, a delicate imaging of the lymphatic system can avoid large incision and intraoperative time-consuming lymphatic vessel dissection. Lymphoscintigraphy used to be the gold standard in evaluating lymphedema and mapping lymphatic systems, but due to the downside of radiation, invasive operation and complication, other modalities are gaining attention and improving the preoperative planning of lymphatic surgeries (4).

We searched the database PubMed and Embase with phrases "lymphovenous anastomosis" "lymphovenous bypass" "LVA", "lymph node transfer" and "LNT". 
Then we reviewed articles within the last 20 years with the topic of "imaging" and "mapping" and nailed the four most commonly reported imaging methods. After that, we scrutinized articles related to the four methods using words "magnetic resonance lymphangiography" "MRL" "indocyanine green" "lymphangiography" "ICG" "ultrasound" "SPECT" and "single photon emission computed tomography computed tomography". For each method, anything we considered as clinical important or innovating is included in this paper.

\section{Indocyanine green (ICG) lymphography}

Since the first use of ICG lymphography by Unno et al. $(5,6)$ for preoperative planning of lyphatico-venous anastomosis, the simplicity, sensitivity, and accuracy has made it a widely used modality in lymphatic imaging (7). Identification of LCVs is essential for a successful LVA. Generally, ICG-enhanced LCVs were considered as functional and ideal for LVA (8-11). This method operates in the nearinfrared region (NIR). ICG is injected preoperatively or intraoperatively, and lymphatic systems can be presented with a good spatial resolution of images (12). However, ICG injection location, depth can affect the outcome. The idea of lymphosome may explain why only lymphatic vessels originating from the injection areas can be imaged (13), superficial lymphatic vessels divide the skin into certain lymphatic territories, in which each vessel drains its own part and does not cross in human. The actual number of "functional" LCVs should take those non-ICG enhanced, but flow-positive ones into consideration (14). Preoperatively ICG-negative lymphatics can also be large and high-flow $(15,16)$. Multi-lymphosome injection may also aid in detecting a greater number of LCVs (17).

For postoperative accessing, ICG lymphography can be used to evaluate the effects of LVA $(18,19)$. Patterns were identified in the patent anastomosis areas. However, for areas that are thick more than $2 \mathrm{~cm}$ like the lower extremity, the limitation of penetration depth made it hard to observe certain dermal backflow when evaluating lymphatic patency rate $(20,21)$. This disadvantage might not be significant in LVA planning for the lymphatic system that was used usually within the depth $<2 \mathrm{~cm}$. Researches on ICG performance in NIR-II $(1,000-1,700 \mathrm{~nm})$ showed that compared with NIR-I window (700-900 nm), it enables superior image quality and increase of image depth $(22,23)$. Indicating a potential clinical broaden use of ICG under future NIR-II hardware.
Recent booming computer techniques also provided new aspects in the utility of ICG. Giacalone et al. reported a case with lymphatic malformation treated with preoperative virtual reality planning (24). Brebant et al. presented an augmented reality intraoperative ICG lymphography navigated LVA (25). These techniques provided surgeons with more intuitive information for preoperative and intraoperative mapping.

In patients with severe lymphedema, lymph node transfer (LNT) was reported to have better relief of symptoms for it reconstructed the damaged or missing lymphatic tissue. ICG was reported to be used in the recipient site for vessel evaluations, vessel patency and perfusion of the transplant $(2,26,27)$. It should be noticed that LNT may result in donor site lymphedema and this could be detected and evaluated by ICG lymphography (28). To reduce the rate of this complication, ICG or Technetium were injected for reverse lymphatic mapping, regarding to reduce the risk of harvesting a draining lymph node (29).

\section{Ultrasound}

Compared with ICG lymphography, ultrasound can detect lymphatic flow in deeper layers which ICG cannot reach, even in regions with stardust or diffuse pattern which would conceal lymphatic vessels in ICG lymphography. Ultrasound device is relatively more commonly used than ICG. These make ultrasound a valuable substitute for ICG lymphography and it has been used in examining lymphedema for years. Three typical ultrasound findings in extremities lymphedema includes increased skin thickness, increased subcutaneous tissue thickness and increased subcutaneous echogenicity (30). Healthy lymphatic vessels showed homogeneous, hypoechoic and specular images under ultrasound, as the develop of lymphatic vessel sclerosis, they would present various shape (31). Hayashi et al. compared ICG lymphography with ultrasound in lower legs LVA and found that ultrasound had a higher detecting rate in identifying lateral side lymphatic vessels, and provide a better distinguish from the surrounding tissues (32). Nevertheless small caliber lymphatic vessels may be hard to distinguish from blood vessels, the use of color doppler mode and tracing origin can help understand the situation (33). In evaluating LNT donor sites, the application in the groin flap showed satisfactory outcome, but the lack of differentiating functional lymph nodes requires the use of other modalities (34).

Same as all other ultrasound implication scenarios, image quality greatly depends on the operators' skill 
and experience. Besides, the accessing process could be time-consuming. Especially in case with massive fluid retention, suggesting a need of decongestive therapy under this circumstance (35). Another difficulty in the use of ultrasound is the caliber of small lymphatic vessel. It is relatively easy to detect dilated lymphatic vessels that are greater than $0.5 \mathrm{~mm}$, but difficult to identify a normal collecting lymphatic vessel that is approximately $0.2 \mathrm{~mm}$, thus the combining use of ICG lymphography (36) and magnetic resonance lymphography (MRL) would to be more accurate $(36,37)$.

\section{MRL}

MRL has proven to be a feasible way to visualize the lymphatic system in patients with lymphedema $(38,39)$, with better spatial and temporal resolution, as well as ability to depict whole extremity lymphatic system. The contrast enhancement within lymphatic vessels and lymph nodes were brought by the subcutaneous injection of paramagnetic macromolecules (>6-10 nm), which entered the lymphatic system and were retained in the lymphatic channels (39).

One main downside about MRL is that it is hard to distinguish lymphatic vessels from blood vessels when there is concomitant venous presence, several techniques were presented to solve this stigma. The caliber, the morphology and the beaded appearance of the vessels can be used in differentiation. Immunohistochemical staining proved it to be effective (40). Adding an MR venogram after the depiction of the lymphatic system could facilitate the differentiation of superficial veins and lymphatic vessels (41). Another solution was to perform a 3D steady-state free precession (SSFP) balanced electrocardiography-(ECG-) triggered sequence (FIESTA, GE) with spectral fat saturation before $3 \mathrm{D}$ gradient-echo T1-weighted MRL, to obtain at the same time the depiction of the severity and distribution of lymphedema and a visualization of a pre-contrast venogram. This method needs well cooperation by the patient for movements would compromise the process (42).

Non-contrast MRL is a noninvasive imaging modality and has been reported in lymphedema evaluation after LNT (43). However, image degradation caused by various biologic motions hampers the use, future hardware and software improvement need to address this (44).

Compared with ICG lymphography and ultrasound, MRL provide the presence, number, course, and location of enhancing lymphatic channels more than $2 \mathrm{~cm}$ deep. With the help of multiplanar reconstruction (MPR) and maximum intensity projections (MIP), 3D images can provide a better understanding of interested region for the surgeon (45) and provide additional information about the surrounding tissue which is helpful to decision making (46,47). Postoperative evaluation using MRL can be performed routinely and with no risk (48). Finding a lymphatic channel with suitable caliber is crucial to the success of surgery, however, the reported size of the vessel can be a lot greater than actual, which emphasizes the need of using both ICG and MRL in preoperative lymphatic mapping (49). Contraindications are the same with all other MR techniques, severe claustrophobia, pace makers, and ferromagnetic intracerebral clips. Complications are rare but not unseen.

\section{Single-photon emission computed tomography- computed tomography (SPECT-CT)}

SPECT-CT is a hybrid imaging examination that could provide morphologic information on computed tomography and functional information on positron emission tomography at the same time (50). While lymocintigraphy demonstrate function of dynamic flow and leave out detailed spatial and anatomical information, SPECT/CT improved by reducing image-degrading effects of partial volume (51). This technique has been used in the preoperative sentinel lymph node imaging in various diseases (52-55). The elaborate depiction of both functional and morphological information was reported to be useful in both LVA and LVNT (56,57). The disadvantages of SPECT-CT include expensive cost and the share of same extra radiation with the plane Tc99 lymphoscintigraphy (57). Preoperative SPECT/CT contains all the necessary information for the surgery. Intraoperative navigation can be realized with the combination of other modalities. Fusion ultrasound provides information from other techniques during the interpretation of ultrasound, when it is linked to preoperative SPECT/CT mapping, real-time image and related anatomical information would provide a reliable navigation through the virtual navigate system (37).

In this paper, we reviewed the most commonly used modalities during the perioperative period of lymphatic surgery (Table 1). However, there are limitations. First, the studies we included are mainly retrospectively studies, therefore, there was a high risk of selection and publication bias. More prospectively double-blind studies are needed. Second, lymphatic surgery is a relatively new technique, at current stage, different imaging modalities have their own 
Table 1 Advantages and disadvantages of different imaging modalities

\begin{tabular}{|c|c|c|c|c|}
\hline $\begin{array}{l}\text { Modalities } \\
\text { ICG Iymphography }(6,7)\end{array}$ & \multicolumn{2}{|c|}{ Advantages } & \multicolumn{2}{|c|}{ Disadvantages } \\
\hline & (II) & $\begin{array}{l}\text { Provide information on location and function of } \\
\text { lymphatic vessels }\end{array}$ & (II) & Contrast medium could cause allergy \\
\hline & (III) & Suitable for intraoperative guidance & & \\
\hline \multirow{2}{*}{ Ultrasound $(32,33,35)$} & (II) & Moderate penetration depth & (II) & Operator dependent \\
\hline & (III) & Suitable for intraoperative guidance & & \\
\hline \multirow[t]{3}{*}{$\begin{array}{l}\text { MR lymphography } \\
(38-40,42)\end{array}$} & $(\mathrm{l})$ & No radiation exposure & (l) & $\begin{array}{l}\text { Time-consuming and patient need to } \\
\text { stay stable through the process }\end{array}$ \\
\hline & (III) & Provide a whole picture of involved extremity & (III) & Contrast medium could cause allergy \\
\hline & (IV) & $3 \mathrm{D}$ reconstruction & & \\
\hline \multirow[t]{3}{*}{ SPECT/CT (53-56) } & $(\mathrm{l})$ & $\begin{array}{l}\text { Comprehensive morphological and functional } \\
\text { information }\end{array}$ & (l) & Time-consuming \\
\hline & (II) & Depiction of the whole body & (II) & Radiation exposure \\
\hline & & & (III) & Expensive cost \\
\hline
\end{tabular}

limits. It is impossible to identify one modality that can fit in all scenarios, therefor, the combining use of proper methods would be more reasonable.

\section{Conclusions}

Application of imaging modalities in lymphatic surgery can be a complex process, involving preoperative staging of lymphedema, mapping, identifying the proper functional lymph vessels, intraoperative navigation, and postoperative evaluation of vessel patency. Choosing the proper means under specific circumstance is crucial to perform a successful operation and result in a satisfactory outcome. The combination use of different modalities provides a compliment to each other and yield a better result. Besides, techniques like fusion imaging, virtual reality, augmented reality and portable devices can add more possibilities in the use of mapping and intraoperative guidance.

\section{Acknowledgments}

Funding: This study was supported by Capital's Funds for
Health Improvement and Research (No. 2016-2-4041), PUMC Youth Fund \& the Fundamental Research Funds for the Central Universities (No.3332015156), to Dr. Chunjun Liu.

\section{Footnote}

Provenance and Peer Review: This article was commissioned by the Guest Editors (Xiaona Lu, Antonio Jorge Forte) for the series "Lymphedema" published in Gland Surgery. The article was sent for external peer review organized by the Guest Editors and the editorial office.

Conflicts of Interest: Both authors have completed the ICMJE uniform disclosure form (available at http://dx.doi. org/10.21037/gs.2020.03.24). The series "Lymphedema" was commissioned by the editorial office without any funding or sponsorship. The authors have no other conflicts of interest to declare.

Ethical Statement: The authors are accountable for all aspects of the work in ensuring that questions related 
to the accuracy or integrity of any part of the work are appropriately investigated and resolved.

Open Access Statement: This is an Open Access article distributed in accordance with the Creative Commons Attribution-NonCommercial-NoDerivs 4.0 International License (CC BY-NC-ND 4.0), which permits the noncommercial replication and distribution of the article with the strict proviso that no changes or edits are made and the original work is properly cited (including links to both the formal publication through the relevant DOI and the license). See: https://creativecommons.org/licenses/by-nc-nd/4.0/.

\section{References}

1. Chang DW, Masia J, Garza R, et al. Lymphedema: Surgical and Medical Therapy. Plast Reconstr Surg 2016;138:209S-18S.

2. Scaglioni MF, Arvanitakis M, Chen YC, et al. Comprehensive review of vascularized lymph node transfers for lymphedema: Outcomes and complications. Microsurgery 2018;38:222-9.

3. Schaverien MV, Badash I, Patel KM, et al. Vascularized Lymph Node Transfer for Lymphedema. Semin Plast Surg 2018;32:28-35.

4. Singhal D, Tran BN, Angelo JP, et al. Technological Advances in Lymphatic Surgery: Bringing to Light the Invisible. Plast Reconstr Surg 2019;143:283-93.

5. Unno N, Inuzuka K, Suzuki M, et al. Preliminary experience with a novel fluorescence lymphography using indocyanine green in patients with secondary lymphedema. J Vasc Surg 2007;45:1016-21.

6. Unno N, Nishiyama M, Suzuki M, et al. Quantitative lymph imaging for assessment of lymph function using indocyanine green fluorescence lymphography. Eur J Vasc Endovasc Surg 2008;36:230-6.

7. Cornelissen AJM, van Mulken TJM, Graupner C, et al. Near-infrared fluorescence image-guidance in plastic surgery: A systematic review. Eur J Plast Surg 2018;41:269-78.

8. Ogata F, Narushima M, Mihara M, et al. Intraoperative lymphography using indocyanine green dye for nearinfrared fluorescence labeling in lymphedema. Ann Plast Surg 2007;59:180-4.

9. Mihara M, Hara H, Narushima M, et al. Lower limb lymphedema treated with lymphatico-venous anastomosis based on pre- and intraoperative icg lymphography and non-contact vein visualization: A case report. Microsurgery
2012;32:227-30.

10. Mihara M, Hara H, Hayashi Y, et al. Upper-limb lymphedema treated aesthetically with lymphaticovenous anastomosis using indocyanine green lymphography and noncontact vein visualization. J Reconstr Microsurg 2012;28:327-32.

11. Suami H, Scaglioni MF. Anatomy of the Lymphatic System and the Lymphosome Concept with Reference to Lymphedema. Semin Plast Surg 2018;32:5-11.

12. Abbaci M, Conversano A, De Leeuw F, et al. Near-infrared fluorescence imaging for the prevention and management of breast cancer-related lymphedema: A systematic review. Eur J Surg Oncol 2019;45:1778-86.

13. Suami H. Lymphosome concept: Anatomical study of the lymphatic system. J Surg Oncol 2017;115:13-7.

14. Yang JCS, Wu SC, Chiang MH, et al. Intraoperative identification and definition of "functional" lymphatic collecting vessels for supermicrosurgical lymphaticovenous anastomosis in treating lymphedema patients. J Surg Oncol 2018;117:994-1000.

15. Narushima M, Yamamoto T, Ogata F, et al. Indocyanine Green Lymphography Findings in Limb Lymphedema. J Reconstr Microsurg 2016;32:72-9.

16. Scaglioni MF, Uyulmaz S, Arvanitakis M, et al. Intraoperatively Detected But Previously Indocyanine Green-Negative Lymphatic Vessels May Have Misprized Potentials and Should Not Be Neglected in Lymphaticovenous Bypass Surgery. Ann Plast Surg 2019;83:69-72.

17. Hara H, Mihara M. Multi-area lymphaticovenous anastomosis with multi-lymphosome injection in indocyanine green lymphography: A prospective study. Microsurgery 2019;39:167-73.

18. Chen WF, Zhao H, Yamamoto T, et al. Indocyanine Green Lymphographic Evidence of Surgical Efficacy Following Microsurgical and Supermicrosurgical Lymphedema Reconstructions. J Reconstr Microsurg 2016;32:688-98.

19. Shih HB, Shakir A, Nguyen DH. Use of Indocyanine Green-SPY Angiography for Tracking Lymphatic Recovery After Lymphaticovenous Anastomosis. Ann Plast Surg 2016;76 Suppl 3:S232-7.

20. Maegawa J, Yabuki Y, Tomoeda H, et al. Outcomes of lymphaticovenous side-to-end anastomosis in peripheral lymphedema. J Vasc Surg 2012;55:753-60.

21. Suzuki Y, Sakuma H, Yamazaki S. Evaluation of patency rates of different lymphaticovenous anastomosis techniques and risk factors for obstruction in secondary upper extremity lymphedema. J Vasc Surg Venous Lymphat 
Disord 2019;7:113-7.

22. Starosolski Z, Bhavane R, Ghaghada KB, et al. Indocyanine green fluorescence in second near-infrared (NIR-II) window. PLoS One 2017;12:e187563.

23. Bhavane R, Starosolski Z, Stupin I, et al. NIRII fluorescence imaging using indocyanine green nanoparticles. Sci Rep 2018;8:14455.

24. Giacalone G, Yamamoto T, Belva F, et al. The Application of Virtual Reality for Preoperative Planning of Lymphovenous Anastomosis in a Patient with a Complex Lymphatic Malformation. J Clin Med 2019. doi: 10.3390/ jcm8030371.

25. Brebant V, Heine N, Lamby P, et al. Augmented reality of indocyanine green fluorescence in simplified lymphovenous anastomosis in lymphatic surgery. Clin Hemorheol Microcirc 2019;73:125-33.

26. Kenworthy EO, Nelson JA, Verma R, et al. Double vascularized omentum lymphatic transplant (VOLT) for the treatment of lymphedema. J Surg Oncol 2018;117:1413-9.

27. Ozturk CN, Ozturk C, Glasgow M, et al. Free vascularized lymph node transfer for treatment of lymphedema: A systematic evidence based review. J Plast Reconstr Aesthet Surg 2016;69:1234-47.

28. Azuma S, Yamamoto T, Koshima I. Donor-site lymphatic function after microvascular lymph node transfer should be followed using indocyanine green lymphography. Plast Reconstr Surg 2013;131:443e-4e.

29. Halvorson EG, Orgill DP. Discussion: reverse lymphatic mapping: a new technique for maximizing safety in vascularized lymph node transfer. Plast Reconstr Surg 2015;135:286-8.

30. Suehiro K, Morikage N, Murakami M, et al. Significance of ultrasound examination of skin and subcutaneous tissue in secondary lower extremity lymphedema. Ann Vasc Dis 2013;6:180-8.

31. Mihara M, Hara H, Kawakami Y. Ultrasonography for classifying lymphatic sclerosis types and deciding optimal sites for lymphatic-venous anastomosis in patients with lymphoedema. J Plast Reconstr Aesthet Surg 2018;71:1274-81.

32. Hayashi A, Yamamoto T, Yoshimatsu H, et al. Ultrasound visualization of the lymphatic vessels in the lower leg. Microsurgery 2016;36:397-401.

33. Hayashi A, Hayashi N, Yoshimatsu H, et al. Effective and efficient lymphaticovenular anastomosis using preoperative ultrasound detection technique of lymphatic vessels in lower extremity lymphedema. J Surg Oncol
2018;117:290-8.

34. Patel KM, Chu SY, Huang JJ, et al. Preplanning vascularized lymph node transfer with duplex ultrasonography: an evaluation of 3 donor sites. Plast Reconstr Surg Glob Open 2014;2:e193.

35. Czedik-Eysenberg M, Steinbacher J, Obermayer B, et al. Exclusive use of ultrasound for locating optimal LVA sites-A descriptive data analysis. J Surg Oncol 2020;121:51-6.

36. Hara H, Mihara M. Usefulness of preoperative echography for detection of lymphatic vessels for lymphaticovenous anastomosis. SAGE Open Med Case Rep 2017;5:2050313X17745207.

37. Gentileschi S, Albanese R, Pino V, et al. SPECT/CT and fusion ultrasound to target the efferent groin lymph node for lymphatic surgery. Microsurgery 2019;39:605-12.

38. Lohrmann C, Foeldi E, Langer M. Indirect magnetic resonance lymphangiography in patients with lymphedema preliminary results in humans. Eur J Radiol 2006;59:401-6.

39. Lohrmann C, Foeldi E, Speck O, et al. High-resolution MR lymphangiography in patients with primary and secondary lymphedema. AJR Am J Roentgenol 2006;187:556-61.

40. Gennaro P, Borghini A, Chisci G, et al. MRL, s-LVA and immunohystochemistry in lymphedema patients. Available online: https://www.europeanreview.org/ wp/wp-content/uploads/687-694-MRL-s-LVA-andimmunohystochemistry-in-lymphedema-patients.pdf

41. Mitsumori LM, McDonald ES, Wilson GJ, et al. MR lymphangiography: How i do it. J Magn Reson Imaging 2015;42:1465-77.

42. Mazzei FG, Gentili F, Guerrini S, et al. MR Lymphangiography: A Practical Guide to Perform It and a Brief Review of the Literature from a Technical Point of View. Biomed Res Int 2017;2017:2598358.

43. Arrivé L, Derhy S, Dlimi C, et al. Noncontrast Magnetic Resonance Lymphography for Evaluation of Lymph Node Transfer for Secondary Upper Limb Lymphedema. Plast Reconstr Surg 2017;140:806e-11e.

44. Arrivé L, Derhy S, El Mouhadi S, et al. Noncontrast Magnetic Resonance Lymphography. J Reconstr Microsurg 2016;32:80-6.

45. Mitsumori LM, McDonald ES, Neligan PC, et al. Peripheral Magnetic Resonance Lymphangiography: Techniques and Applications. Tech Vasc Interv Radiol 2016;19:262-72.

46. Zeltzer AA, Brussaard C, Koning M, et al. MR 
lymphography in patients with upper limb lymphedema:

The GPS for feasibility and surgical planning for lymphovenous bypass. J Surg Oncol 2018;118:407-15.

47. Kim EY, Hwang HS, Lee HY, et al. Anatomic and Functional Evaluation of Central Lymphatics With Noninvasive Magnetic Resonance Lymphangiography. Medicine (Baltimore) 2016;95:e3109.

48. Lohrmann C, Felmerer G, Foeldi E, et al. MR lymphangiography for the assessment of the lymphatic system in patients undergoing microsurgical reconstructions of lymphatic vessels. Microvasc Res 2008;76:42-5.

49. Pons G, Clavero JA, Alomar X, et al. Preoperative planning of lymphaticovenous anastomosis: The use of magnetic resonance lymphangiography as a complement to indocyanine green lymphography. J Plast Reconstr Aesthet Surg 2019;72:884-91.

50. Israel O, Pellet O, Biassoni L, et al. Two decades of SPECT/CT - the coming of age of a technology: An updated review of literature evidence. Eur J Nucl Med Mol Imaging 2019;46:1990-2012.

51. Weiss M, Baumeister RGH, Frick A, et al. Primary lymphedema of the lower limb: the clinical utility of single photon emission computed tomography/CT. Korean J Radiol 2015;16:188-95.

52. Krzhivitskii PI, Novikov SN, Kanaev SV, et al. The use of single-photon emission computed tomography-computed tomography in detecting multiple metastatic lymph nodes in patients with breast cancer. Nucl Med Commun

Cite this article as: $\mathrm{Du} \mathrm{X}, \mathrm{Liu} \mathrm{C}$. Application of imaging in lymphedema surgical therapies. Gland Surg 2020;9(2):582-588. doi: $10.21037 / g s .2020 .03 .24$
2019;40:169-74.

53. Naumann CM, Colberg C, Jüptner M, et al. Evaluation of the diagnostic value of preoperative sentinel lymph node (SLN) imaging in penile carcinoma patients without palpable inguinal lymph nodes via single photon emission computed tomography/computed tomography (SPECT/ CT) as compared to planar scintigraphy. Urol Oncol 2018;36:92.e17-92.e24.

54. Remenschneider AK, Dilger AE, Wang Y, et al. The predictive value of single-photon emission computed tomography/computed tomography for sentinel lymph node localization in head and neck cutaneous malignancy. Laryngoscope 2015;125:877-82.

55. McGregor A, Kim S, Norwich-Cavanaugh A, et al. Clinical Value of Single-photon Emission Computed Tomography Combined With Computed Tomography for Sentinel Lymph Node Identification in Melanoma. Ann Plast Surg 2019;82:S192-4.

56. Iimura T, Fukushima Y, Kumita S, et al. Estimating Lymphodynamic Conditions and Lymphovenous Anastomosis Efficacy Using (99m)Tc-phytate Lymphoscintigraphy with SPECT-CT in Patients with Lower-limb Lymphedema. Plast Reconstr Surg Glob Open 2015;3:e404.

57. Dionyssiou D, Demiri E, Sarafis A, et al. Functional lymphatic reconstruction with the "Selected Lymph Node" technique guided by a SPECT-CT lymphoscintigraphy. J Surg Oncol 2019;120:911-8. 